\title{
Criminologie
}

\section{Critique de la justice criminelle}

\section{G. A.B. Watson}

Volume 11, numéro 2, 1978

Normes et politique criminelle

URI : https://id.erudit.org/iderudit/017089ar

DOI : https://doi.org/10.7202/017089ar

Aller au sommaire du numéro

Éditeur(s)

Les Presses de l'Université de Montréal

ISSN

0316-0041 (imprimé)

1492-1367 (numérique)

Découvrir la revue

Citer cet article

Watson, G. A. (1978). Critique de la justice criminelle. Criminologie, 11(2), 6-24. https://doi.org/10.7202/017089ar d'utilisation que vous pouvez consulter en ligne.

https://apropos.erudit.org/fr/usagers/politique-dutilisation/ 
L'intérêt que suscite actuellement la question des normes de justice criminelle tient non pas à ce qu'on pense qu'il se produit une dégradation générale du processus de la justice criminelle en tant que telle mais plutôt à ce qu'on est de plus en plus sensible à l'impression, notamment, qu'elle est en retard par rapport à l'évolution de la société vers un nouveau degré de conscience d'elle-même et de son autodétermination. Du fait de cette évolution, de nombreuses institutions et professions sont soumises à un examen qui, sans être hostile, est certainement critique. En effet, il y a relativement peu de pratiques et de relations traditionnelles qui soient encore tenues pour acquises par ceux qui s'y trouvent assujettis. Le souci qui commence à se manifester au sujet des droits individuels peu importe leur nature, c'est-à-dire qu'il s'agisse des malades hospitalisés, des travailleurs, des prisonniers, des femmes, des enfants, des accusés, des membres de groupes minoritaires, etc., consiste avant tout dans la recherche d'une juste protection contre les abus du pouvoir. On refuse la pression des institutions traditionnelles de la société et on participe au mouvement en vue de modifier la répartition de ce pouvoir, non pas nécessairement pour saper les institutions elles-mêmes, mais de façon à élever la condition de ces gens, de celle de sujets ou de victimes, à celle de participants actifs, sinon de partenaires, qui ont de l'initiative, de la dignité et une maîtrise de leur propre destinée.

L'appareil de la justice criminelle n'est donc pas seul mis en cause en ce qui a trait à ses postulats, son efficacité, son mode d'action, ses attitudes et ses pratiques, bien qu'il soit peut-être parmi les derniers à faire l'objet d'une enquête systématique. Il ne faudrait pas penser non plus que c'est seulement, ou même surtout, qu'on s'en préoccupe de l'extérieur. Au contraire, les critiques les plus informées et tranchées viennent de l'intérieur même du système et leurs auteurs comptent parmi ceux dont le rôle serait le plus sérieusement atteint par le changement. Cela a été démontré lors du colloque sur les «Normes d'application de la loi $\gg$, tenu au Centre de criminologie de Toronto en 1978, auquel ont participé plusieurs représentants de la justice criminelle canadienne. On abonde également dans le même sens aux 
États-Unis dont dernièrement lors d'une conférence sur les progrès de la justice criminelle parrainée par le National Clearing for Criminal Justice Planning and Architecture de la Law Enforcement Assistance Administration.

La majorité des citoyens canadiens seraient fort étonnés sans doute de constater combien certaines de ces critiques sont graves et combien elles portent sur presque chaque aspect de la justice criminelle. D'une manière générale, on soutient que l'appareil ne fonctionne pas du tout dans son ensemble, qu'il y a trop peu de coordination entre les activités de ses divers éléments, que l'entente et que les communications mutuelles sont insuffisantes. On signale, en fait, les tensions et les rivalités, sinon les conflits qui existent entre les divers secteurs qui travaillent de façon isolée, chacun étant sur la défensive et craignant que son action soit sapée par l'attitude et les pratiques des autres.

On soutient également que de toute façon, la justice criminelle fonctionne d'une façon qui n'est guère comprise, appréciée ni respectée par l'ensemble de la collectivité tandis qu'elle-même n'a guère le sentiment d'appartenir au tissu social ordinaire et moins encore de lui être comptable. Les membres de la profession juridique, les policiers et les agents de correction se disent souvent éloignés de la vie des gens ordinaires alors que le système dans son ensemble est accusé par ses propres membres d'être, entre autres choses, " inhumain », illogique et inefficace, isolé, confus et dénué du sentiment qu'il a un but et un rôle gaspilleur, capricieux, nuisible et lui-même, discriminatoire - et marqué par les disparités régionales et juridictionnelles.

Tous les éléments sont visés par ces accusations et il s'en ajoute d'autres qui concernent les activités et les structures particulières qui caractérisent chacun. L'application de la loi, d'ordinaire assimilée au rôle de la police, tend à être sujette aux critiques les plus nombreuses parce que, sans doute, c'est l'elément le plus étendu et le plus exposé aux regards du public. Cependant, ici encore, les préoccupations relatives à son action existent tout autant à l'intérieur qu'en dehors du monde policier. On peut examiner ces critiques particulières dans le cadre de diverses catégories.

1) La definition même de la fonction : elle est confuse, contentieuse constamment sujette à changer. La fonction est défi- 
nie et évaluée de différentes façons par des éléments différents de la collectivité et de la justice criminelle et, en fait, par les organes d'application de la loi eux-mêmes. Cela étant, on ne semble guère chercher à faire l'unanimité.

2) Les relations avec d'autres parties du système : les critiques d'ordre général ont été indiquées plus haut, mais la police, en particulier, exprime des préoccupations au sujet de la magistrature, de la conduite des procès et de l'existence (ou de l'inexistence) de coutumes en ce qui concerne la condamnation. Autant de problèmes qui, à ses yeux, semblent n'avoir guère de rapport avec les réalités de la rue, la nature (ou la sous-culture) criminelle, les circonstances de tel crime en particulier et les événements qui entourent les enquêtes et qui indiquent qu'on ne comprend guère la nature de l'activité policière. Beaucoup de policiers sont frappés par ce qui leur paraît être un manque total de responsabilité de la part de la magistrature tandis que leur propre corps est constamment appelé à rendre compte de ses actes devant le public, la profession juridique, la magistrature et les politiciens.

3) Les relations avec la collectivité sont en grande partie caractérisées par un sentiment d'aliénation qui frise souvent l'hostilité, là encore, il y a une indifférence et un manque de compréhension à l'égard des problèmes liés à la fonction policière de l'application de la loi ainsi qu'une apparente abdication des responsabilités du public en cette matière.

4) L'organisation, la communication et la gestion du personnel au sein des services eux-mêmes : il s'agit là de questions relatives à la distribution des ressources, la division du travail, les lignes de commandement, le recrutement, la sélection, la formation, la motivation, l'estime, la promotion, la planification de la carrière, les soins de santé et la consultation, pour n'en nommer que quelques-unes qui, souvent, semblent être laissées au hasard d'une personnalité particulière qui occupe un poste de commande, ou aux circonstances liées à la taille et à la situation géographique d'une localité.

\section{Les normes}

Étant donné les préoccupations générales et diffuses que suscite la justice criminelle, une des premières questions à soule- 
ver est celle des normes. Lorsqu'un acte, simple ou complexe, semble souftrir d'insuffisances d'une nature générale ou chronique, l'explication facile consiste à supposer un manque de normes ou une insuffisance en ce qui concerne leur niveau. Le terme "normes " est, bien entendu, ambigu. Il peut signifier simplement des «attentes 》. Relever ces normes c'est relever les attentes. Le terme peut signifier des \& exigences \- comme c'est le cas lorsque ces attentes deviennent des commandements, ou des obligations. Il peut signifier des buts, ou des objectifs plus ou moins importants et constants pour une personne, ou un groupe de gens. Les normes sont aussi des unités de mesure et de comparaison. A ce titre, elles permettent de porter des jugements, surtout des jugements de valeur, des appréciations, etc. L'absence d'une norme, ce qui signifie qu'il n'existe aucun moyen d'évaluer une chose, indique souvent qu'on ne se soucie pas de la qualité, du but et de l'efficacité. Les normes peuvent être simplement descriptives d'objets ou d'actions d'ordre particulier et complexe.

Elles se rattachent pour autant à la définition d'une chose. \& Pour être tel et tel, il faut qu'il y ait telle et telle chose, et ainsi de suite. $\gg \mathrm{Ne}$ pas avoir de norme en ce sens, c'est ne pas savoir en quoi consiste une chose et, partant, ne pas avoir de moyens de la reconnaître et encore moins de l'évaluer.

En bref, les normes assurent un moyen de reconnaître, d'évaluer et d'utiliser. Elles fournissent une orientation et un moyen de contrôler un objet ou une activité, peu importe leur simplicité ou leur complexité. Quand un système fonctionne mal, il y a quelque chose qui ne va pas dans les commandes, ou une partie est en panne; dans les deux cas, le diagnostic et le traitement obligent à appliquer des normes.

\section{Application de la loi}

Si le terme de «normes » est ambigu, la notion d'application de la loi est vague. L'expression évoque à coup sûr une idée de force et fait penser aussi à des sujets résistants qui sont contraints d'obéir aux agents de la loi ou à un élément souverain qui la dicte. Ce n'est pas là, naturellement, une conception jugée appropriée au Canada, bien qu'elle comporte manifestement un certain élément de vérité. Une vue plus appropriée adoucit et étend le terme s application » pour $\mathrm{y}$ inscrire les pressions modérées exercées pour assurer le respect de la loi, y compris en fin 
de compte l'enseignement et l'éducation dans une société libre et démocratique. Peu importe la méthode d'application, si elle réussit, la loi n'est pas enfreinte, ce qui signifie, dans le cas du droit criminel, qu'il y a prévention de la criminalité. Il faudrait donc envisager la prévention de la criminalité comme étant l'essence même de l'application de la loi. Il est intéressant de noter que l'expression signifie, dans l'esprit des gens, une activité qui s'exerce après la perpétration du crime - sa détection et l'appréhension du délinquant. C'est là, il va de soi, la partie passionnante et celle qui se prête à la rédaction de bons récits pleins d'aventures. C'est cependant le côté négatif de l'application de la loi qui absorbe, en fait, moins de $20 \%$ du temps et de l'énergie de la police.

Dans l'esprit du public, l'application de la loi est pour ainsi dire synonyme d'action policière (une autre preuve que l'application de la loi est perçue en termes de correction, de sévérité), mais, dans la mesure où une société s'éloigne d'un système autocritique et despotique, l'application de la loi devient une responsabilité publique et générale, tout comme les lois ellesmêmes sont devenues des instruments servant à assurer le bienêtre public et social. Nous y reviendrons par la suite.

\section{La loi et les moyens d'application}

Si l'objet de la loi, dans une démocratie moderne, consiste à réglementer le comportement en vue du bien-être de la société, son efficacité à cet égard dépend autant de la façon dont elle est édictée, que des principes qui l'ont inspirée, des moyens qui président à son application et du contenu de lois particulières, si importantes soient-elles. En d'autres termes, les normes d'application sont aussi importantes que celles que les lois elles-mêmes cherchent à consacrer. En fait, elles devraient, en principe, être identiques, sinon l'entreprise ne rime à rien et la notion d'application de la loi est pour ainsi dire en contradiction avec ellemême tout autant lorsqu'on édicte des lois qui ne peuvent être appliquées.

Si, dans l'administration de la justice, nous souscrivons au Canada aux idéaux d'humanité, d'uniformité et d'efficacité, aucun acte d'application ne devrait les contredire ni en principe, ni sur des points particuliers. Il n'est pas nécessaire non plus que l'un soit sacrifié à la longue au profit des autres, bien qu'il semble 
souvent à court terme que tous ne peuvent se réaliser simultanément. Des situations comme celles-là sont celles qui revêtent un caractère déchirant dans la pratique de l'application de la loi et c'est ici que les organes d'application de la loi ont besoin de directives les plus claires et de l'appui le plus grand d'une société prête à comprendre et à accepter les conséquences de ses propres idéaux. Des situations de cette nature sont aussi celles qui exigent le plus de discrétion, ce qui est un autre domaine de préoccupation.

\section{Études antérieures sur les normes d'application de la loi}

La question des normes de justice criminelle, et en particulier d'application de la loi, est à l'ordre du jour depuis plusieurs années au Canada, comme aux États-Unis et à l'étranger. On a à cet égard, réalisé une somme énorme de travaux puisque chaque aspect de la question a été étudié, discuté et exposé. Aux ÉtatsUnis, par exemple, deux grandes opérations se dégagent de nombreux travaux qui ont eu un rôle important dans la pensée actuelle, soit les travaux de la National Advisory Commission on Criminal Justice Standards and Goals et ceux de l'American Bar Association sur les normes de justice criminelle. Il s'agit dans les deux cas de travaux d'ordre à la fois général et détaillé qui traitent d'une foule de données devant être examinées attentivement par quiconque s'intéresse à la question et cela dans n'importe quel contexte national.

En ce qui concerne l'application de la loi, le groupe d'étude sur la police de la National Advisory Commission a présenté un rapport de 670 pages qui portait sur les buts, la fonction, les programmes et l'efficacité et énonçait environ 120 "normes » connexes aux principaux aspects de l'activité policière. Le rapport traitait aussi des relations de la police avec la justice criminelle dans son ensemble et avec la collectivité en général et rejoignait en cela, la plupart des préoccupations actuelles du milieu canadien.

On peut en dire autant du rapport de l'American Bar Association sur les « Normes relatives à la fonction policière urbaine, de la National Advisory Commission. Les responsables des forces policières canadiennes connaissent ces documents américains. Ils y ont réfléchi à la lumière de la situation qui existe chez nous et y ont sans doute contribué, au moins indirectement, 
par les nombreux contacts qu'ils ont avec leurs homologues d'outre-frontière.

Une enquête sur les normes constitue incontestablement le premier pas vers un examen sérieux de la justice criminelle en général et de l'application de la loi en particulier. Il en est ainsi pour diverses raisons. Une telle enquête transforme les critiques relativement diffuses, impressionnistes et individuelles à l'égard d'incidents particuliers en une entreprise plus systématique et constructive qui vise les conditions générales présentes dans les diverses institutions. En vertu de l'ambiguïté même de la notion de normes, l'enquête passe des problèmes immédiats et manifestes aux questions plus fondamentales mais moins évidentes dont on doit traiter dans toute tentative d'évaluation des méthodes et procédures devenues la pratique quotidienne de la justice criminelle.

Aux problèmes immédiats et évidents correspondent d'ordinaire d'immédiates et évidentes solutions. Pour corriger $A$, il faut faire B. Mais pourquoi corriger A ? Qu'y a-t-il de mal chez A ? La réponse se référera à $\mathrm{C}$ qui est un objectif ou une norme. On ne peut chercher des solutions valables que lorsque cela est clair.

Ainsi, par exemple, si l'objectif des organes d'application de la loi est de réduire la criminalité, une norme de progrès orienté vers la réalisation de cet objectif consistera dans une certaine réduction de la criminalité. Pour atteindre cette norme (réduire la criminalité), les opérations de l'organe doivent en arriver à une certaine norme d'efficacité et cela par l'adoption de mesures destinées à améliorer le rendement. Ces mesures, en tant que règles générales, deviennent des normes de procédure dans la multitude des activités et des décisions que comporte l'action policière. Elles nous font descendre sur le plan des détails, soit des critères de sélection et des programmes des cours de formation, d'une part, à la composition et aux pouvoirs des commissions de la police, d'autre part. Pour des raisons d'ordre pratique, les études antérieures sur l'application de la loi se situent en bas de l'échelle par rapport aux questions ultimes, c'est-à-dire parmi les questions de détail, questions sur lesquelles porte principalement la bibliographie.

Il est donc manifeste que nous possédons déjà, quant aux questions de détail de toute action policière, une mine de textes 
sur les normes. Des centaines, pour ainsi dire, de points ont fait l'objet d'un examen. Les organes d'application de la loi qui désirent améliorer leur action n'ont qu'à étudier la bibliographie et à choisir parmi les excellentes suggestions qui s'y trouvent les mesures, les procédures et les critères les mieux adaptés à leur situation locale.

Fait intéressant, cependant, cette tâche apparemment simple n'apparaît nulle part comme la solution, du moins non pas comme la solution principale. La raison en est que ce ne sont pas des questions de détail qui constituent le problème ultime. Certaines autorités policières craignent, non sans un certain scepticisme bien compréhensible, que cette préoccupation au sujet de normes d'efficacité opérationnelle n'ait d'autre effet que de replâtrer l'institution, sauf si l'on agit à la lumière de buts et de fonctions bien déterminés qui assureront le contexte de ce que nous entendons par efficacité et le moyen de mesurer le succès lorsque nous l'aurons obtenu. Il n'est pas de trésor de normes qui puisse servir l'agent de police qui n'a pas un ensemble bien clair de priorités et une conception fondamentale, appuyée par son milieu communautaire, de son rôle au sein de la société, ainsi que de son lien avec ses collègues dans l'appareil de la justice criminelle. C'est cela qui semble manquer aux yeux de ceux qui sont le plus en contact avec la justice criminelle et c'est à ce problème qu'aboutissent toutes les discussions sérieuses de la question des normes.

Dans les propos qu'il a tenus au Colloque sur les normes de l'application de la loi, le surintendant en chef Roy Moffat, directeur du Collège canadien de police, a dit :

La définition de normes relatives à l'activité policière est une tâche complexe parce qu'elle suppose une définition bien claire du rôle de la police. Or, je ne crois pas que ce rôle soit bien défini; au contraire, les efforts menés en vue de bien le déterminer ont été infructueux. Ce qui s'en est dégagé, ce sont les problèmes liés à la fonction policière, et non la solution de ces derniers. Dans le contexte canadien, qui ne diffère pas tant d'autres milieux, le rôle de la police a été forgé à dessein et par défaut. L'élaboration de normes ne devrait-elle pas se pencher d'abord sur le rôle de la Police ?...

Il ajoutait :

Cette cuvre devrait conduire à se demander quels sont les objectifs de l'application de la loi. Comprennent-ils seule- 
ment l'application des lois par la détection, l'appréhension et la poursuite des infracteurs ou leur portée s'étend-elle à des questions de prévention et de service. Des normes plus précises qui reflètent toute la mission de la police - prévention de la criminalité, protection de la vie humaine et des biens, détection et appréhension des criminels et maintien de l'ordre public - s'imposent peut-être.

L'introduction du rapport de l'American Bar Association faisait valoir le même point, mais en mettant l'accent sur un autre aspect. Au sujet de l'ensemble des «normes » proposées par l'Association, il y est dit :

Très peu sont énoncées dans des termes qui en font des procédures modèles susceptibles de s'appliquer immédiatement sur une base nationale. Ces normes représentent plutôt, pour la plupart, une approche à suivre pour faire face aux problèmes et aux besoins qui confrontent... les corps policiers.

Cette utilisation variée des « normes » est dans une grande mesure le reflet du caractère sous-développé du domaine policier. Il importe beaucoup plus aujourd'hui de reconnaître de façon plus générale les réalités et complexités de l'activité policière (par exemple, que la police constitue un important organe administratif chargé d'exercer une vaste discrétion) que de s'entendre sur la mécanique du fonctionnement d'un corps policier (par exemple, que les agents de police mesurent 5 pi. et 6 po. et soient d'un poids proportionné à leur taille). Le fait, cependant, qu'on accorde si peu d'attention aux grands problèmes de fond empêche d'en parler avec la précision avec laquelle on peut traiter des préoccupations plus traditionnelles connexes au fonctionnement d'un service de police. Il faut espérer, cependant, que ces normes susciteront de vastes discussions, provoqueront de nouvelles tentatives en vue de cerner des besoins et programmes particuliers à la police dans tout le pays et, finalement, joueront un rôle important dans l'application des nombreux changements si nécessaires pour permettre à la police de bien exercer sa fonction dans la société.

En outre, l'expérience de ceux qui ont effectué ces enquêtes porte à croire que le processus d'enquête, qui fait graduellement la lumière sur ce dont il s'agit réellement dans la justice criminelle et l'application de la loi, est plus utile que la compilation de normes spécifiques qui s'en dégagent. Cela n'a rien d'étonnant. Comme tous les phénomènes qui, en dernière analyse, dépendent des actes humains, leur succès ultime dépend plus de la qualité 
des objectifs et de la clarté et de la conviction avec lesquelles ils sont perçus que de l'efficacité des moyens adoptés pour les réaliser.

Dire ici que la recherche de normes dans des questions de détail peut marquer le pas afin que l'attention des décisionnaires puisse se porter sur des questions plus fondamentales, ce n'est pas affirmer que l'appareil de la justice criminelle peut se relâcher dans ses efforts en vue d'améliorer son action à tous égards pendant qu'on discute de ces graves questions. Cela signifie que l'examen de ces questions ne peut plus être différé. Cela signifie aussi qu'il faut procéder à cet examen avec un soin et une discipline extraordinaire parce qu'il s'agit d'un sujet exposé, de par sa nature, à une foule de dangers.

Nous allons essayer d'inscrire ces questions «préalables , dans un certain ordre et de donner une indication de leur à propos et des liens qui les unissent. En procédant du général au particulier, on aboutit aux points suivants :

- application de la loi et philosophie politique ;

- responsabilité ;

- application de la loi et éducation du public ;

- participation des citoyens ;

- relations au sein de l'appareil de la justice criminelle ;

- relations entre les instances policières ;

- organisation policière.

\section{Application de la loi et philosophie politique}

Parce que l'examen des buts fait ressortir des valeurs et des engagements d'ordre personnel et collectif, il n'est pas étonnant non plus qu'il s'entreprenne avec répugnance et que le processus d'enquête et d'étude soit tout aussi menaçant que les critiques qui l'ont amorcé. Une fois commencée, cette enquête obéit à sa logique interne qui la conduit à contester sans arrêt chaque postulat. Une question aussi simple, par exemple, que celle de savoir quel est le rôle de la police dans la société nous pose la question préalable bien évidente qui est de savoir de quel genre de société il s'agit. Nous sommes fort conscients de la distinction entre ce que nous appelons la \& société démocratique \& et l' État policier ». Que l'idée de police soit au cœur de cette distinction montre bien l'importance du sujet. Quand nous considérons, ensuite, la question de la nature de l'\&application de la loi $\gg$, le 
même genre d'autres questions se posent, c'est-à-dire : quelle est la loi qui est appliquée, à l'égard de qui, par qui et par quels moyens ? Cela nous amène de nouveau à la nature de la société. L'emploi, en réponse à de telles questions, des termes tels que l' Etat» ou le «souverain » ne fait que mettre en lumière la complexité du sujet et indique qu'on ne peut l'écarter comme étant purement académique.

En effet, de la réponse à cette question dépendent des décisions touchant d'autres questions : reddition de comptes, source et limite de l'autorité et du pouvoir et, en fin de compte, responsabilité de la détermination du rôle de la police. Même si les différences entre les deux extrêmes de société que nous appelons démocratique et, mettons, totalitaire, dans ces questions d'organisation mettent en cause l'éthos, les valeurs fondamentales et le sens de sa valeur personnelle de chaque membre, elles se reflètent dans les moindres détails des interactions humaines par le truchement des attitudes qu'elles suscitent.

En outre, dans la distinction entre société démocratique et société totalitaire, les lois qui existent dans chacune sont moins importantes que la façon dont elles sont édictées et appliquées. L'État totalitaire est capable d'édicter de très «bonnes 》 lois, mais selon la notion démocratique, la vertu d'une loi particulière est anéantie par le mal de la sujétion, par le truchement de cette loi, à la volonté de quelqu'un qui commande de sa propre initiative et au pouvoir physique qui l'applique. Par contre, selon l'idéal démocratique, chaque citoyen a le droit et le devoir de partager la responsabilité de l'élaboration des lois et de leur application, même si certaines lois peuvent être «mauvaises » et si toutes sont appliquées de façon insuffisante.

Certes, il est rare qu'un système se présente dans la pratique sous ses formes pures, mais le premier pas à faire, quand il est question de normes, est de décider quel système doit être l'idéal. Le pas suivant, ou plutôt la tâche subséquente, consiste à voir à ce que chaque mesure et chaque procédure visent à réaliser cet idéal et à montrer le modèle qu'il assure. La constitution et les formalités de notre système parlementaire le font, mais, audessous de cet échelon, il en est laissé beaucoup plus aux contingences de la nature humaine où deux grands facteurs exercent leur action de façon contradictoire : le désir du pouvoir et le désir d'éviter les responsabilités. 
Dans un état d'équilibre égal, ces deux facteurs peuvent s'annuler ; cependant, quand ils sont pondérés comme à l'ordinaire, la majorité des gens sont contents de se soustraire aux responsabilités et une minorité tient à exercer le pouvoir. Cet état de choses ne favorise pas la réalisation des idéaux de la démocratie, mais plutôt les conditions propices à un État policier.

Même si une société adopte la démocratie comme un idéal avoué, elle peut aboutir par défaut à quelque chose qui ressemble plus à l'Etat policier, et cela du fait de sa paresse et de son indifférence ou, pourrait-on dire simplement, de son immaturité politique.

La première catégorie de normes devrait énoncer le modèle politique et les caractères essentiels du système de justice criminelle qui en découle.

\section{Responsabilité}

Si le modèle doit être le modèle démocratique et si son essence consiste dans la participation du public à l'autogouvernement, la responsabilité vitime de l'élaboration des lois, ainsi que de leur application incombe alors aux membres de la société, auquel cas il s'ensuivrait que l'apparition de fonctions spécialisées - légiférer, administrer et appliquer - tient simplement aux nécessités pratiques de la vie sociale complexe qui exigent une division du travail afin que les choses se fassent bien. S'il en est ainsi, ces responsabilités particulières et les pouvoirs connexes sont délégués aux personnes choisies à cette fin par lo public (dont les talents particuliers et le temps serviront à la poursuite d'autres fins). Il faudrait noter à ce propos que, même si l'ordre, la paix et la sécurité publics sont choses précieuses en elles-mêmes dans tous les contextes politiques, leur principale raison d'être en régime démocratique sera de maintenir un milieu où la société peut prospérer dans la poursuite de l'épanouissement personnel au bénéfice de chacun de ses membres.

Si tel doit être le modèle, la délégation et la réception de responsabilités et de pouvoirs spéciaux comportent un échange de confiance et d'engagement qui constituera la base de l'exercice du pouvoir. Personne ne s'en trouve dégagé de responsabilités, ni nanti de pouvoirs sans bornes et tous les exécuteurs spéciaux de cet ordre en deviennent agents du public et comptables envers 
lui. Chaque élément de la justice criminelle devrait manifester ce fait, une fois adoptée la décision principale.

Il semble bien que dans plus d'une démocratie « de l'ouest » qui se prétend telle c'est là une décision qui, pour dire le moins, est tenue en suspens comme si, après tout, ce n'était peut-être pas le modèle sur lequel nous nous réglons, ou que cette décision fondamentale n'était pas prise avec conviction. Si tel est le cas, il faudrait le reconnaître et il faudrait faire face à la question avant de s'occuper de normes.

En outre, certains jugements des tribunaux établissent de fortes réserves touchant la situation du modèle démocratique dans notre société. Parlant de la question de la responsabilité, au récent colloque de Toronto, $\mathbf{M}$. W. Shimmin, conseiller auprès de la Commission de police d'Ontario, a signalé, par exemple, qu'étant donné les assurances formulées en 1829 par sir Robert Peel à l'effet que la police et le public sont précisément dans la même situation par rapport à la loi et sont conjointement tenus d'en assurer le maintien, deux décisions récentes de la Cour d'appel d'Ontario niaient explicitement l'existence d'une relation de maître et serviteur entre le public et la police. Il cite le juge Laidlaw qui, en 1957, déclarait :

La vraie situation d'un agent de police est énoncée comme il suit par le vicomte Simonds, dans A.G. de N.S.W. c. la Perpetual Trustee Co. (Ltd.), 1955, A.C. 489.

Il existe une différence fondamentale entre la relation domestique d'un serviteur et d'un maître et celle du titulaire d'une charge publique et de l'État qu'il est dit servir.

Le juge Laidlaw (parlant au nom de la cour plénière) a ajouté :

Son autorité est primitive et non déléguée et il l'exerce à sa discrétion en vertu de sa charge. C'est un agent ministériel qui exerce des droits statutaires indépendamment d'un contrat (Renvoi aux termes de la loi sur les questions constitutionnelles Johnson c. la ville de Grimsby, 28 O.R., 1957, Cour d'appel).

Il signale ensuite la décision plus récente encore qu'a rendue la Cour d'appel et qui se lit comme suit :

...l'agent de police a le droit de toucher son salaire, non en échange ou en considération des fonctions qu'il exerce, mais plutôt du fait qu'il est titulaire de la charge (Mahood 
c. la Commission de police de la région d'HamiltonWentworth et la Municipalité régionale d'Hamilton-Wentworth. N'a pas fait l'objet d'un compte rendu, fév. 1977).

De tels jugements font réféchir quand on est tenté de décrire, en termes trop simples, les fonctions de la justice criminelle et les corrélations qui existent entre elle et le public. La question fondamentale subsiste toujours, cependant, et il faut y répondre en termes clairement compris de tous et capables de bien se traduire par une action concrète. Quand quelque chose d'analogue au volume, par habitant, des actions civiles intentées contre les corps policiers et les municipalités à l'heure actuelle aux Etats-Unis existera au Canada, il se peut bien que la question de la responsabilité ultime aura été au moins en partie réglée, même si peu de gens comprendront probablement au début que celui qui doit rendre des comptes est responsable.

Une catégorie de normes devrait porter sur les circuits de responsabilité.

\section{Application de la loi et éducation du public}

Peu importe que la relation entre la police et le public en soit une de serviteur-maître ou autre, comme nous l'avons dit, l'application de la loi devient de plus en plus une responsabilité publique que les citoyens s'imposent à mesure qu'on s'éloigne du modèle totalitaire. Plus la société se fait populeuse et complexe, plus le maintien de la loi dépend de l'initiative du public. Il n'est pas d'efficacité chez un corps policier qui puisse compenser le désintérêt et l'irresponsabilité d'une collectivité. Une catégorie de normes devrait se rattacher à l'aptitude réelle de la société à exercer ses responsabilités, c'est-à-dire à accepter les conséquences de ses propres exigences en fait de paix et de sécurité, d'ordre et de liberté.

Cela nous amène à la question de l'éducation. L'application de la loi commence au foyer et se poursuit à l'école. C'est ici que notre attention devrait se fixer afin de rendre la société plus consciente de la nature de la liberté et de l'autogouvernement dans sa manifestation quotidienne et pratique. Les questions de loi, de gouvernement, de liberté, de responsabilité, de devoir, de droits, etc. n'occupent pas à l'heure actuelle, une place importante dans l'expérience scolaire. En fait, à l'école et au foyer, ces questions comptent avec l'éducation sexuelle parmi les éléments 
les plus fortuits et cavaliers de l'éducation des enfants. Une bonne partie de cette conscientisation consiste à rendre explicite ce qui est implicite dans les formalités mystérieuses du système. Il s'agit en grande partie d'amener régulièrement les divers organes d'administration de la justice dans la salle de classe et souvent dans le cadre de l'enseignement normal. Le fait d'envoyer un agent de relations publiques faire la tournée des écoles deux fois par année n'influe pour ainsi dire aucunement sur l'attitude des enfants. L'éducateur entreprenant et sérieux peut trouver beaucoup à faire dans ce domaine.

\section{Participation des citoyens}

On pourrait dire que les normes des trois catégories susdites jettent les bases des décisions à adopter au sujet de l'organisation et des procédures de l'action quotidienne de ce que nous appelons l'application de la loi. Il semble bien jusqu'ici que la recherche actuelle de normes ne saurait se séparer du mouvement social général vers une autodétermination plus poussée et une répartition, ou un partage plus étendu des pouvoirs. Quand cette tendance s'affirme, elle suppose bien clairement une rupture avec une tradition qui s'en remet, pour l'exercice du pouvoir, à une attitude distante, à la mystification et à l'intimidation. Cette tendance cherche plutôt à remplacer la crainte du système et des fonctionnaires par le respect fondé sur la compréhension et sur un sentiment d'investissement personnel dans ses fins et ses méthodes.

Le dernier pas dans cette voie serait une certaine forme de participation des membres de la collectivité à l'établissement des principes et à la planification de l'application de la loi, à la définition du rôle de la police, à la fixation de normes et même au choix et à la formation des agents de police et à l'administration interne des organes d'application eux-mêmes.

Ainsi donc, une quatrième catégorie de normes porterait sur la participation officielle du public, ou de la collectivité à la fonction policière, c'est-à-dire sur leur rôle au sein de commissions de police, sur la liaison entre la commission et d'autres corps municipaux, sur le rôle et la composition des commissions de police provinciales, et sur la participation des citoyens au processus de sélection et aux questions de formation et de personnel. 
D'autre part, ce qui est également important, c'est la participation formelle de la police à d'autres fonctions au sein de la collectivité, telles que l'enseignement, les services de santé, les loisirs, l'urbanisme et l'affectation des terrains, etc.

La question, une fois de plus, est celle du rôle de la police, c'est-à-dire où tirer la ligne entre le spécialiste et le généraliste et qui décide où il faut la tracer.

\section{Relations au sein de l'appareil de la justice criminelle}

Un examen du rôle de l'application de la loi, qui ne s'inscrit pas dans une étude d'ensemble de la justice criminelle, accentuerait les inquiétudes qui s'expriment déjà en ce qui a trait à celle-ci en tant qu'un vrai système, et en fausserait de toute façon, les conclusions. Les questions en jeu sont à la base, nous l'avons vu, de la notion même de la loi ainsi que de l'application de la loi et de la répression des manquements de la loi. La division entre la justice civile et la justice criminelle est déjà assez artificielle elle-même sans qu'il faille fragmenter davantage le processus.

Comment la relation entre la police, les tribunaux et les services de correction doit se comprendre en théorie et se manifester dans la pratique constitue un problème difficile en luimême, mais c'en est un qui a une portée directe et profonde sur les fonctions respectives de ces éléments. Dans l'examen d'un de ces éléments, il faut faire intervenir la voix des autres afin que la clarification des rôles et des corrélations et l'orientation de la philosophie fondamentale tiennent compte des réalités et des intérêts de tous les éléments qui sont liés ensemble. Cela se traduira par une cinquième catégorie de normes à l'échelon des principes.

Les avantages d'ordre instructif assurés aux participants à cette sorte d'entreprise justifieraient en eux-mêmes le temps et l'énergie dépensés. Ces avantages ont des répercussions dans tout le système. Ici encore, cependant, le groupe devrait comprendre de soi-disant profanes, des citoyens d'en dehors de l'appareil de la justice criminelle, ainsi que des gens qui ont été pris dans le système en tant qu'accusés, victimes, témoins et jurés. 
Relations entre les instances policieres

La catégorie suivante de normes régirait la relation entre les diverses instances et les forces policières. $\mathrm{Si}$ nous soutenons que l'application de la loi est un continuum à l'intérieur du tissu de la société, il est contradictoire de maintenir un système de divisions de compétences qui laisse des lacunes et des incongruités dans l'ensemble de la fonction policière. Il y a la G.R.C., les sûretés provinciales et les sûretés municipales. Il existe des corps policiers privés. Il est manifeste qu'il faut en arriver à l'uniformité et à la cohérence dans les normes de toute la profession. Il faut aussi respecter les différences qui sont appropriées. Ni l'un ni l'autre ne sont possibles sans une bonne coordination de la fonction et une supervision générale. Cependant, la coordination et la supervision de toute la gamme des responsabilités policières supposent deux choses. Tous les types d'organes d'application de la loi doivent figurer dans l'étude comme sujets et participants et dans tout organisme de supervision subséquemment établi.

Au surplus, il faut aussi s'arrêter à la grande question de la participation à la supervision. On reproche aux commissions de police actuelles d'être insuffisamment représentatives des groupes intéressés et abusivement représentatives de certains groupes particuliers. Il importe plus, semble-t-il, d'y figurer que d'en être exclus et cela devrait à coup sûr signifier la représentation du public profane dans plus d'une catégorie (y compris la jeunesse), des grands éléments de l'organisation policière (gendarmes, personnel de formation et autres membres de la police en plus des cadres supérieurs) et d'autres éléments de la justice criminelle, y compris l'industrie de la police privée.

\section{Organisation policière}

Les questions que nous avons évoquées et les tendances que nous avons notées interviennent dans les rouages internes des organes d'application de la loi eux-mêmes où elles constituent, tout aussi manifestement, une rupture avec les traditions du modèle militaire de la police et inclinent plutôt vers de nouvelles structures d'autorité, de responsabilité et de participation. Les corps policiers sont déjà bien avancés sur la voie de l'abandon du modèle militaire grâce, en particulier, à l'établissement d'associations policières qui constituent presque des unités de négociation. 
C'est peut-être le jalon de mi-chemin, soit la rupture, de l'unique chaîne de commandement à sens unique, en deux éléments : patron et ouvriers. Il existe maintenant deux adversaires, ce qui est débilitant dans une certaine mesure, mais les travailleurs sont actifs plutôt que passifs et veulent intervenir davantage dans les décisions. Le mouvement se continue dans le cadre du grand phénomène social et ne devrait vraisemblablement pas s'arrêter et encore moins se renverser. Au fur et à mesure que la police obtiendra l'exercice d'un rôle toujours plus responsable à tous les échelons et dans toutes les décisions, les implications en fait de normes de procédures en seront plus profondes.

Rien n'indique que de telles tendances amènent un abaissement des normes (c'est-à-dire de la qualité) d'action, ou qu'elles nuisent à la discipline ou à l'efficacité. Elles constituent une menace pour certaines des formes particulièrement superficielles du comportement, du décorum et des relations traditionnels, bien que ce soit la mise en question de ces formes qui fait partie de l'évolution étant à la base des efforts de changements actuels. Ces tendances rendent l'administration de la police plus complexe et font jouer de nouvelles forces et des critères nouveaux dans les décisions et la communication. Elles se répercutent donc directement sur les types de personnalités qui sont capables de fonctionner dans ces conditions différentes et sur ceux qui ne le peuvent pas. Souvent, la personnalité et le style des personnes constituées en autorité décident du sort de propositions de changements destinés à instaurer un style de police qui serait déplaisant. C'est un problème habituel qui, aux yeux de ceux qui cherchent à expérimenter et à changer les choses, fait en quelque sorte que ce sont les inférieurs qui mènent les supérieurs.

Les normes relatives à l'organisation interne, tout en reflétant le stade où en sont les tendances importantes à un moment particulier, devraient aussi en prévoir l'orientation. Plus encore que cela, cependant, elles devraient représenter des initiatives et des décisions au sujet des questions fondamentales qui déterminent la nature de la profession policière comme carrière. Les idées de profession et de carrière fixent elles-mêmes une norme d'attente pour ceux qui sont dans la police et ceux qui en sont à l'extérieur. Une bonne partie du travail relatif aux normes consiste donc à accorder la pratique aux idées courantes, qu'il s'agisse de la formation, de l'affectation des ressources, de la 
promotion, etc., ou de l'élaboration des décisions et de la communication.

\section{Normes d'application de la loi et recherche criminologique}

Une bonne partie des propos sur les questions de normes, de rôles et de principes en matière de justice criminelle sont d'ordre idéologique et impressionniste. Ces propos se fondent en grande partie sur l'expérience personnelle particulière et sur des anecdotes transmises par une fervente tradition orale. Quand on commence à discuter, il n'y a pas beaucoup de contraintes qui s'exercent pour orienter les propos et pour en authentifier les conclusions - si tant est qu'on en vienne à des conclusions.

L'élément le plus important, en plus de voix intelligentes et expérimentées, c'est l'information dont elles disposent. Cette information doit être fournie par une judicieuse recherche qui seule permettra de séparer les données concrètes des impressions et des conjectures.

Le Centre de criminologie de Toronto a trouvé chez la police, les avocats, les tribunaux, les victimes et les accusés un remarquable empressement à participer à une recherche on ne peut plus exigeante ; recherche qui révélera à tous, de l' intérieur \& comme de l' «extérieur », les réalités de la justice criminelle - ses points forts aussi bien que ses points faibles - et dont les conclusions amèneront finalement des améliorations pour tout le monde. Cette manifestation de bonne volonté est un signe de santé et d'intérêt. Il faudrait l'accueillir et l'exploiter à plein aux fins qu'elle vise et il faudrait qu'elle intervienne pleinement dans l'examen dont il est question ici. Amorcer cet examen sans les données que, sauf quelques exceptions, les éléments de la justice criminelle sont prêts à fournir à des chercheurs sérieux, constitue un geste irresponsable. 